Jakobovits, J., Wiggins, E. H. \& Harrison, J. S. (1951). J. gen. Microbiol. 5, 648-656.

\title{
The Effect of Optically Active Forms of Some Amino-Acids on the Response of Yeast to $\beta$-Alanine and Pantothenic Acid
}

\author{
By J. JAKOBOVITS, E. H. WIGGINS AND J. S. HARRISON \\ The Distillers Company Ltd., Research and Development Department, \\ Great Burgh, Epsom, Surrey
}

SUMMARY: The growth-promoting action of $\beta$-alanine on Saccharomyces carlsbergensis and two strains of $S$. cerevisiae is stimulated or inhibited to different extents by the optical enantiomorphs of methionine, glutamic acid, asparagine and $\alpha$-alanine. This behaviour can be considered as due, in each individual case, to one or more of three separate effects: $(a)$ stimulatory, $(b)$ inhibitory, and $(c)$ antagonistic. In general, the L-forms of the amino-acids have a more pronounced action than the D-isomers. Racemic mixtures have intermediate effects. Similar but less striking results are observed when $\beta$-alanine is replaced by pantothenic acid. Atypical effects are obtained with $\mathrm{D}$-alanine and $\mathrm{D}$-glutamic acid.

Amino-acids exert an inhibitory effect on the growth of certain organisms in the presence of $\beta$-alanine, as shown by Sarett \& Cheldelin (1945) and others. Hartelius (1946) found that L-glutamic acid and L-asparagine had a depressant action on the growth of yeast when the ratio $\alpha$-amino-acid : $\beta$-alanine was higher than a critical value, below which a stimulatory effect was observed. In order to obtain more information concerning the mode of interference of $\alpha$-amino-acids with the metabolism of $\beta$-alanine, the effects of both the Dand L-forms of a number of amino-acids have been studied and compared with the action of the corresponding DL-mixtures.

Previous workers have described several instances where D-amino-acids inhibited the growth of micro-organisms, while the L-isomers were inert. Thus, Davis \& Maas (1949) found that inhibition by DL-serine of the growth of Escherichia coli was due to D-serine only. Similarly Mueller \& Miller (1949) demonstrated that $\mathbf{D}$-serine inhibited the formation of tetanus toxin. It was therefore expected that the action of the optical enantiomorphs on $\beta$-alanine would be unequal, and that, in general, the effect of the racemic form would be intermediate between the two. Results given in this paper support this idea.

\section{METHODS}

Growth Tests

Response curves were obtained by growing yeast in defined culture media containing graded amounts of $\beta$-alanine or calcium $D$-pantothenate and plotting dose against growth response. Control curves with no addition of $\alpha$-amino-acid were compared with others after addition of fixed concentrations of D-, L- and DL-amino-acids.

Organisms and inocula. The test organisms were Saccharomyces carlsbergensis 4228, and two strains of $S$. cerevisiae. These were maintained in a liquid culture medium containing $16 \%(\mathrm{w} / \mathrm{v})$ malt extract, transfers being 
made to malt wort-agar slopes $24 \mathrm{hr}$. before the test was commenced. The inoculum was prepared by suspending a loop of yeast from a slope in saline, measuring the yeast content of this suspension by means of a Spekker absorptiometer, and diluting with saline to a concentration of $100 \mu \mathrm{g}$. moist yeast $/ \mathrm{ml}$.

Medium. The double-strength basal medium was essentially the same as that described by Atkin, Schultz, Williams \& Frey (1943), except that pyridoxine hydrochloride replaced calcium pantothenate, and casein hydrolysate was omitted. It had the following composition: (g.) glucose, 40; potassium citrate, 20 ; citric acid, $4 ;\left(\mathrm{NH}_{4}\right)_{2} \mathrm{HPO}_{4}, 2 ; \mathrm{KH}_{2} \mathrm{PO}_{4}, 1 \cdot 1 ; \mathrm{KCl}$, $0 \cdot 85 ; \mathrm{CaCl}_{2}, 0 \cdot 25 ; \mathrm{MgSO}_{4} .7 \mathrm{H}_{2} \mathrm{O}, 0 \cdot 25 ;$ (mg.) $\mathrm{MnSO}_{4} .4 \mathrm{H}_{2} \mathrm{O}, 5 ; \mathrm{FeCl}_{3}, 5$; inositol, 50 ; nicotinic acid, 5 ; pyridoxine $\mathrm{HCl}, 5$; aneurin, 0.5 ; $(\mu \mathrm{g}$.) biotin, 16 ; water to 1 l., the $\mathrm{pH}$ being adjusted to 5.0 with $\mathrm{HCl}$ or $\mathrm{NaOH}$. A further modification, introduced by Hopkins \& Pennington (1947), was the addition of a quantity of nicotinic acid equal in weight to the pyridoxine hydrochloride.

Procedure. Double-strength medium $(5 \mathrm{ml}$.) was distributed in $50 \mathrm{ml}$. conical flasks. Into each of one series of flasks, used as standards, $\beta$-alanine (or calcium pantothenate) solution $(2 \mathrm{ml}$.) was pipetted, the series of flasks containing in a typical experiment $0 \cdot 25,1,4,16$ and $64 \mu \mathrm{g}$. of $\beta$-alanine (or the molar equivalent of calcium pantothenate); $2 \mathrm{ml}$. of water were then added. Three other series of flasks contained, instead of water, $2 \mathrm{ml}$. of D-, L- or DL-amino-acid solution, usually $4 \mathrm{mg} . / \mathrm{ml}$. Duplicate flasks were used in all cases. To each of one set of flasks, serving as blanks, were added $5 \mathrm{ml}$. of medium and $4 \mathrm{ml}$. of water, so that a check could be made on the absence of $\beta$-alanine from the medium. All flasks were plugged with cotton wool and sterilized at $5 \mathrm{lb} . / \mathrm{sq}$. in. for $15 \mathrm{~min}$. After cooling and adding $1 \mathrm{ml}$. of inoculum, the flasks were shaken at a frequency of 100 strokes $/ \mathrm{min}$. through a horizontal distance of $1 \mathrm{in}$. for $17 \mathrm{hr}$. in an incubator at $30^{\circ}$. The inoculum and growth period were so chosen that the highest growth obtained was less than half the maximum possible in the medium used.

Measurement of growoth and plotting of results. After incubation 1 drop of $10 \%$ sodium azide was added to check growth, followed by $20 \mathrm{ml}$. of cold water; after mixing, the light transmission of the resultant yeast suspension was measured with a Spekker absorptiometer using neutral grey filter H508, and the readings were converted to mg. dry weight of yeast by means of a calibration curve. The duplicate results did not generally differ by more than $3 \%$. The weight of yeast dry matter in milligrams was plotted against the $\beta$-alanine or pantothenic acid concentrations on double-logarithmic graph paper.

\section{Note on the resolution of amino-acids}

As the D-forms of the amino-acids were not commercially available the racemic mixtures were resolved as follows:

Methionine. The enzymic method of Brenner \& Kocher (1949), based on the fact that pancreatin will hydrolyse the isopropyl ester of $\mathrm{L}$-methionine, but not of D-methionine, was strictly followed. 
Glutamic acid. Essentially Fischer's (1899) method of resolving benzoylDL-glutamic acid with strychnine was used.

Asparagine. DL-Asparagine was allowed to crystallize slowly from water at room temperature. Well-shaped crystals of the active enantiomorphs were formed and these were separated mechanically by observing the shape of the crystal faces.

a-Alanine. DL-Alanine was benzoylated as described by Levy \& Palmer (1942), and the benzoyl derivative was resolved with strychnine and brucine (Pacsu \& Mullen, 1940).

\section{RESULTS AND DISCUSSION}

The results obtained with $S$. carlsbergensis are presented graphically in Figs. 1, 2 and 3. Similar responses obtained with both strains of $S$. cerevisiae indicated that there was no significant difference between the behaviour of the three yeasts under the experimental conditions described. Preliminary experiments were carried out to determine the most suitable range of doses. The graphs given are those for the regions of most characteristic effects. Internal replication in the tests was good, and in all cases smooth curves were obtained.

It is clear from the experimental evidence that the extent to which $\alpha$-aminoacids stimulate or inhibit the response of yeast to $\beta$-alanine depends upon their

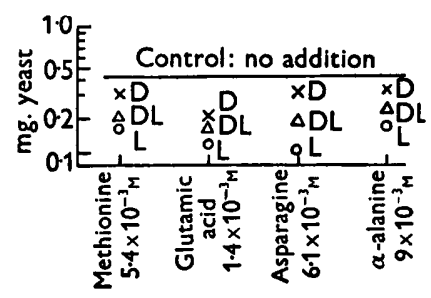

Fig. 1. Effect of D-, L- and DL-amino-acids on the response of S. carlsbergensis in the absence of pantothenic acid and $\beta$-alanine.

optical configuration. All four acids studied depressed to a marked degree such growth as is obtained in the complete absence of $\beta$-alanine or pantothenic acid (Fig. 1). The L-acids are more effective than the $D$-isomers, with the DL-forms intermediate. At concentrations of $\beta$-alanine or pantothenic acid of $0.28 \times 10^{-6} \mathrm{M}$, when the proportion of $\alpha$-amino-acid to growth factor was highest, the effects were in general similar. Methionine (Fig. 2A and B) depressed growth at all concentrations of pantothenic acid or $\beta$-alanine tested, and the relative effect of the enantiomorphs of this amino-acid remained unchanged with increasing concentration of growth factor. In another experiment not recorded, the isomers of methionine had the same relative inhibitory effects when the concentration of $\beta$-alanine was increased to $5 \cdot 5 \times 10^{-3} \mathrm{M}$. In the experiments with glutamic acid (Fig. 2C and D) and asparagine (Fig. 2E and F) a second effect was involved at higher 
concentrations of $\beta$-alanine and pantothenic acid: in the presence of the L-amino-acids there was greater growth than in the standards.

D-Alanine (Fig. 2G and $\mathrm{H}$ ) gave growth which was almost equal to the standard, suggesting that there may be some stimulatory action. This was
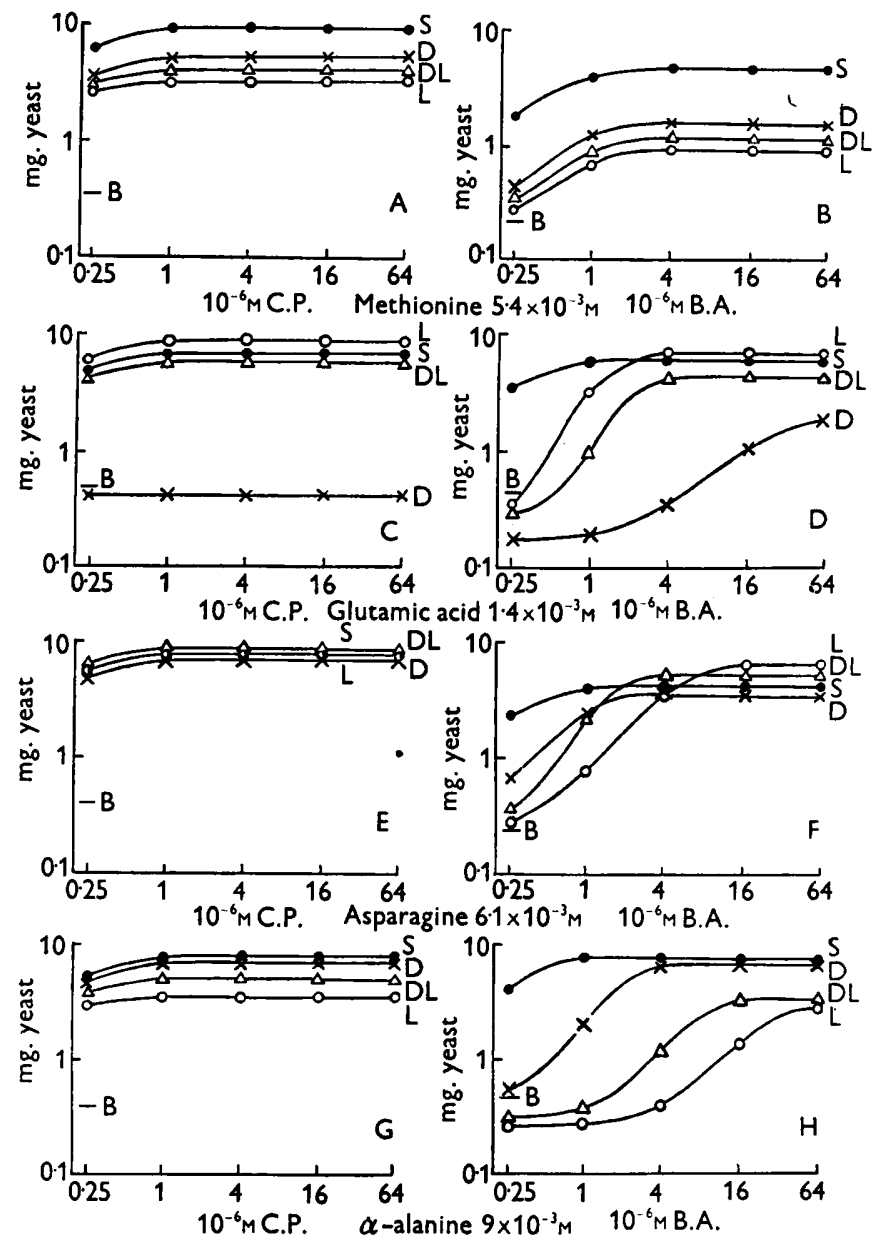

Fig. 2A-H. Effect of D-, L- and DL-amino-acids on the response of $S$. carlsbergensis to calcium pantothenate and $\beta$-alanine. C.P., calcium D-pantothenate; B.A., $\beta$-alanine; S, standard with no added $\alpha$-amino-acid; $B$, blank for standard curve with no added calcium pantothenate or $\beta$-alanine.

confirmed by the fact that in one of the strains of $S$. cerevisiae there was a definite increase of growth above the standard. L-Alanine had a growthdepressant action even at relatively high concentrations of $\beta$-alanine, but at the highest levels this effect was largely overcome. The effect produced by $\mathrm{D}$-alanine was similar, but only a sixfold increase of $\beta$-alanine concentration was required to produce the same growth as the standard, whereas the corresponding ratio for the L-isomer was sixty. 
In view of these facts, it seems necessary to distinguish between three distinct effects of the $\alpha$-amino-acids.

(1) Inhibitory effect. The first and simplest case is illustrated in Fig. 2A and $\mathbf{B}$, where the addition of $5 \cdot 4 \times 10^{-3} \mathrm{M}$ methionine depressed growth in the presence of both pantothenic acid and $\beta$-alanine to approximately the same extent throughout the range of concentrations. Graphically this is expressed by the fact that the $\log : \log$ curves obtained on addition of methionine were below, and roughly parallel to, the standard curves. This would indicate that both the $\mathrm{D}$ - and L-forms of methionine had a depressant effect on the yeast growth, which was probably independent of the metabolic processes connected with $\beta$-alanine or pantothenic acid, the $\mathrm{L}$-isomer being more active than the $\mathrm{D}$-form. The effect can be considered as due to an unspecified toxic action on the yeast cells which may be connected with competitive inhibition of amino-acid or protein synthesis.

(2) Stimulatory effect. The second effect is shown in Fig. $2 \mathrm{c}$, where L-glutamic acid stimulated growth above the standard. Probably the stimulatory effect was also independent of processes connected with the utilization of pantothenic acid or its synthesis from $\beta$-alanine, because it was constant at all higher doses of both growth factors, i.e. in complete medium. The effect is possibly concerned with nitrogen assimilation or protein synthesis (Thorne, 1949; White \& Munns, 1950).

The stimulatory effect of the natural or L-isomers is not surprising, but the observation that $\mathrm{D}$-alanine stimulated yeast growth is more difficult to explain. Snell and co-workers (Holden, Furman \& Snell, 1949; Holden \& Snell, 1949) observed that $D$-alanine could replace vitamin $\mathbf{B}_{6}$ for the growth of certain lactic acid bacteria. Growth of yeast in pyridoxine-free medium, with and without $\mathrm{D}$-alanine, did not reveal any connexion between the two factors; in fact, using one strain of $S$. cerevisiae, D-alanine was stimulatory to the same extent at all levels of pyridoxine, pyridoxal or pyridoxamine in the presence of an optimal concentration of pantothenic acid, which indicates that the effect was independent of vitamin $\mathbf{B}_{6}$. It can only be suggested that D-alanine is required for some reaction within the cell and cannot be synthesized sufficiently rapidly to allow the yeast to grow at its maximum rate.

(3) Antagonistic effect. The third condition is illustrated in Fig. 2H, which shows the effect of $\alpha$-alanine in the presence of $\beta$-alanine. This action of $\alpha$-alanine (described above) is represented graphically by typically hysteresisshaped curves. This type of response is characteristic of the action of a metabolic antagonist, and is taken to express the fact that an enzyme concerned in the chemical processes connected with the formation or function of the growth factor is blocked by a compound of similar molecular structure when present in large excess, but as the ratio of antagonist to growth factor decreases (i.e. at high concentrations of growth factor) the inhibitory effect is overcome and growth approaches normal. In this case, the enzyme concerned can most easily be visualized as that which catalyses the reaction by which $\beta$-alanine combines with pantoic acid to form pantothenic acid, because the antagonistic effect was never observed in the presence of pantothenic acid 
alone. $\alpha$-Alanine, being chemically similar to $\beta$-alanine, might be expected to have a strong antagonistic action, and it is striking that in these experiments, the greatest antagonistic effect was observed with this amino-acid. Further, in view of many similar observations by workers in this field, the natural L-isomer would be most likely to have the more pronounced effect. As noted above, the inhibition ratio of L-alanine to $\beta$-alanine was only about a tenth of that of $\mathrm{D}$-alanine to $\beta$-alanine. Similar, but smaller, effects were shown by L-glutamic acid (Fig. 2D) and by L-asparagine (Fig. 2F) in the presence of $\beta$-alanine. In these cases only about four times the usual concentration of $\beta$-alanine for optimal growth was required to overcome the antagonistic effect. If it be accepted that the strong antagonistic action of $L$-alanine to the growth of yeast, in the presence of $\beta$-alanine, but not pantothenate, is due to the structural similarity of the $\alpha$ - and $\beta$-forms, an explanation of the antagonistic effects of L-glutamic acid and L-asparagine may be sought in the conversion of these amino-acids to $\alpha$-alanine by the reactions in which asparagine is converted to aspartic acid by asparaginase, and aspartic and glutamic acids to $\alpha$-alanine by transaminases. These enzymes are specific for the L-isomers of the amino-acids.

Combinations of the inhibitory, stimulatory and antagonistic effects described above would explain all the responses obtained, except that of D-glutamic acid, which is discussed below. The effects on the response to pantothenic acid of D- and L-methionine (Fig. 2A), and L-alanine (Fig. 2G), are examples of inhibition only. D- and L-Methionine (Fig. 2B), and D-asparagine (Fig. 2F) had a similar action in the presence of $\beta$-alanine. D-and L-Alanine had a clear-cut antagonistic effect in the presence of $\beta$-alanine (Fig. $2 \mathrm{H}$ ), the action of the L-isomer being stronger than that of the $\mathrm{D}$-form. This action was combined with a small inhibitory effect throughout the entire range, and in the case of the D-isomer, a slight stimulatory effect was superimposed at high concentrations of $\beta$-alanine. L-Glutamic acid (Fig. 2c) had a stimulatory action on the response to pantothenic acid, except at very low levels of pantothenic acid (Fig. 1). L-Glutamic acid (Fig. 2D) and L-asparagine (Fig. 2F) both had a stimulatory effect on the response to $\beta$-alanine, upon which was superimposed an antagonistic action.

The case of D-glutamic acid was extraordinary in that growth was strongly inhibited throughout the full range of pantothenic acid used (Fig. 2C), whereas L-glutamic acid stimulated, and the DL-mixture had an action very similar to that of the L-isomer. On the other hand, in the presence of $\beta$-alanine (Fig. 2D), the inhibitory effect of the $\mathrm{D}$-form was decreased at high levels of $\beta$-alanine. This observation was confirmed by carrying out a special experiment in order to exclude all variables such as different inocula, and slight differences in temperature or time of incubation. Fig. 3 illustrates the effect of varying the concentration of D-glutamic acid on the response to $\beta$-alanine. It will be seen that with increasing amounts of glutamic acid the effect is qualitatively similar, but greater in degree. At first sight this behaviour was unexpected, because if $\beta$-alanine be converted to pantothenic acid before it can exert a growth-factor effect, it would be expected that the response to $\beta$-alanine 
would be depressed to as great an extent as the response to pantothenic acid. However, the following explanation of the mechanism is presented in view of further experimental evidence.

It has been shown that pantothenic acid can form a conjugate with glutamic acid, presumably in the L-form (King \& Cheldelin, 1948; King, Fels \& Cheldelin, 1949), and it is possible that such a compound must first be formed before pantothenic acid can function as a growth factor. In such a case, D-glutamic acid might be expected to be an antagonist to the enzyme system which effects the conjugation. Evidence for this was obtained by using mixtures of the $\mathrm{L}$ - and $\mathrm{D}$-forms in varying proportions in tests with pantothenic acid. It was found that a small proportionate addition of the L-isomer would

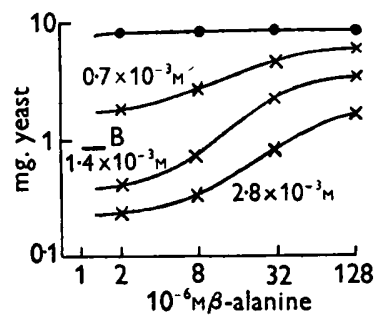

Fig. 3. Effect of varying concentrations of D-glutamic acid on the response of $S$. carlsbergensis to $\beta$-alanine; $\mathbf{B}$, blank for standard curve with no added calcium pantothenate or $\beta$-alanine.

neutralize the depressant effect of the $D$-form, suggesting a competitive inhibition of the action of the enzyme, which has a much stronger affinity (of the order of fiftyfold) for the L-acid than for the $\mathrm{D}$-isomer. Further investigation showed that three different $\mathrm{D}$-amino-acids (methionine, asparagine and $\alpha$-alanine) added in the ratio of 1 part amino-acid to 9 parts of $\mathrm{D}$-glutamic acid (total weight $2 \mathrm{mg}$./flask) had no significant effect in removing the inhibition, whereas similar additions of the $\mathrm{L}$-forms, and of glycine and $\beta$-alanine which do not contain an asymmetric carbon atom, removed the inhibition almost as effectively as L-glutamic acid. Thus it is concluded that at lower levels $\beta$-alanine functions as a growth factor, being converted into pantothenic acid, but that at high levels it performs a second function in relieving the inhibitory effect of D-glutamic acid, and therefore produces the characteristically-shaped curve of Fig. 2D, as compared with the total inhibition in the presence of pantothenic acid only (Fig. 2c).

Various workers have suggested mechanisms for the inhibitory effect of $\alpha$-amino-acids on $\beta$-alanine metabolism. Nielsen, Hartelius \& Johansen (1944) considered that the following two reactions were competing:

$$
\begin{aligned}
\beta \text {-alanine + pantoic acid } & \leftrightharpoons \text { pantothenic acid, } \\
\text { glutamic acid +pantoic acid } & \leftrightharpoons \text { pantoylglutamic acid. }
\end{aligned}
$$

Glutamic acid would, therefore, tend to decrease the amount of pantothenic acid that could be formed. Later Hartelius (1946) proposed a further side 
reaction in which a dipeptide was formed from $\beta$-alanine and the interfering amino-acid:

$$
\beta \text {-alanine }+ \text { glutamic acid } \leftrightharpoons \text { dipeptide. }
$$

The dipeptide was assumed to be inactive, i.e. it could not form an active pantoyl compound. Neither of these processes would account for inhibition when pantothenic acid replaces $\beta$-alanine. It would seem better, therefore, to postulate that inhibition is caused primarily through competitive interference by the inhibitor with certain specific side chains of the active enzyme molecules, with a consequent displacement of the substrate. Such inhibition is discussed by Rydon (1948) in greater detail.

A diversity of opinion exists on the subject of stimulation. Ehrlich's and Stickland's work in this field is well known and has been summarized by Thorne (1949). It has been suggested by Thorne, and also by White \& Munns (1950) that in certain circumstances amino-acids are assimilated intact by growing yeast. This explanation would only cover the stimulatory effect of single amino-acids if it were assumed that the majority of the amino-acids could be synthesized sufficiently rapidly to have no restricting effect on yeast growth, whilst a small number, such as glutamic acid and asparagine, could not be formed at this rate. This can only be partially true at best, because the addition of all the amino-acids known to be required by yeast results in a faster rate of growth than is produced by the few which are stimulatory alone. At present the experimental evidence is insufficient to enable exact conclusions on the mechanism of inhibition or stimulation to be drawn.

The authors thank the Directors of the Distillers Company Ltd. for permission to publish this paper.

\section{REFERENCES}

Atkin, L., Schultz, A. S., Williams, W. L. \& Frey, C. N. (1943). Yeast microbiological methods for determination of vitamins. Pyridoxine. Industr. Engng. Chem. Anal. Ed. 15, 141.

Brenner, M. \& Kocher, V. (1949). Eine einfache enzymatische Methode zur Herstellung von D- und L-Methionin. Helv. Chim. Acta, 32, 333.

Davis, B. D. \& MaAs, W. K. (1949). Inhibition of $E$. coli by D-serine and the production of serine-resistant mutants. J. Amer. chem. Soc. 71, 1865.

Fischer, E. (1899). Utber die Spaltung einiger racemischer Amidosäuren in die optisch-activen Componenten. Ber. dtsch. chem. Ges. 32, 2451.

Hartelius, V. (1946). Glutamic acid, aspartic acid, asparagine and glutamine as anti-growth substances for $\beta$-alanine. C.R. trav. Lab. Carlsberg, Sér. physiol. $24,185$.

Holden, J. T., Furman, C. \& Snell, E. E. (1949). D-Alanine and the vitamin Bo content of micro-organisms. J. biol. Chem. 178, 789 .

Holden, J. T. \& SNell, E. E. (1949). The relation of $D$-alanine and vitamin $B_{6}$ to the growth of lactic acid bacteria. J. biol. Chem. 178, 799.

Hopkins, R. H. \& Pennington, R. J. (1947). The assay of the vitamin $B_{0}$ complex. Biochem. J. 41, 110.

Krng, T. E. \& Cheldelin, V. H. (1948). Pantothenic acid studies. II. The influence of glutamic acid and pantoic acid on yeast growth. Arch. Biochem. 16, 231.

King, T. E., Fels, I. G. \& Cheldelin, V. H. (1949). Pantothenic acid studies. VI. A biologically-active conjugate of pantothenic acid. J. Amer. chem. Soc. 71, 131. 
Levy, M. \& Palmer, A. H. (1942). The benzoylation and resolution of alanine. J. biol. Chem. 146, 493.

Mueller, J. G. \& Muller, P. A. (1949). Inhibition of tetanus toxin formation by D-serine. J. Amer. chem. Soc. 71, 1865.

Nielsen, N., Hartelius, V. \& Johansen, G. (1944). Über die Antiwuchsstoffe der Pantothensäure und des $\beta$-Alanins. C.R. trav. Lab. Carlsberg, Sér. physiol. 24, 39.

Pacsu, E. \& Mullen, J. W. (1940). An improved method for the resolution of synthetic alanine. J. biol. Chem. 136, 335.

Rydon, H. N. (1948). D-Amino-acids in microbiological chemistry. Biochem. Soc. Symposia No. 1, p. 40, Cambridge University Press.

Saret, H. P. \& Cheldelin, V. H. (1945). The utilisation of $\beta$-alanine and pantothenic acid by yeasts. J. Bact. 49, 31.

Thorne, R. S. W. (1949). Nitrogen metabolism of yeast. A consideration of the mode of assimilation of amino-acids. J. Inst. Brew. 55, 201.

White, J. \& MunNs, D. J. (1950). Utilisation of aspartic acid and asparagine by yeast. Nature, Lond., 165, 111.

(Received 31 July 1950) 